\title{
LA COMERCIALIZACIÓN POTENCIAL DE LA PAPA AMARILLA EN LA REGIÓN JUNÍN*
}

\author{
Limas Amorin, César y y Gómez Herrera, John²
}

Facultad de Industrias Alimentarias de la Universidad Nacional del Centro del Perú

\begin{abstract}
RESUMEN
La investigación documenta la forma de comercialización de la papa amarilla de la región Junín en mercados locales y extranjeros, identificando, analizando y optimizando la oferta, la demanda existente y latente para un producto que es utilizado por su alto valor nutricional; el estudio de costos de producción y venta del producto nos permitirá incrementar y desarrollar estrategias de comercialización. El Perú como país de origen de la papa, cuenta con una gran biodiversidad de este tubérculo, produciendo el $72 \%$ de las variedades que existen a nivel mundial. La importancia de este cultivo a nivel internacional es comprobada, siendo el cuarto alimento básico en la dieta de la población mundial, contribuyendo a reducir el déficit alimenticio, más aún en un contexto donde los principales bienes sustitutos muestran signos de escasez, como es el caso del arroz. Los productores de papa a nivel nacional no son homogéneos, varían entre el sector más integrado a los mercados de bienes hasta los agricultores de autoconsumo. Se necesita la interacción de los agricultores, instituciones promotoras, inversionistas, el Estado para desarrollar la rentabilidad y beneficios en función de la demanda estimada; considerando un tamaño ideal de producto, proceso de transformación, mercado, precio, envase, promoción del producto y publicidad, es decir, papa fresca, seleccionada, clasificada, limpia, lavada, empacada y con marca, aprovechando las ventajas competitivas y comparativas de la papa amarilla peruana; interesando a los inversionistas nacionales o extranjeros, ampliando a nuevos mercados.
\end{abstract}

Palabras clave: papa amarilla, oferta, demanda, promoción, publicidad, rentabilidad.

\section{THE POTENTIAL COMMERCIALIZATION OF THE YELLOW POTATO OF THE JUNÍN REGION}

\begin{abstract}
The research documents the commercialization way of the yellow potato of the Junin region in local and foreign markets, identifying, analyzing and optimizing the offer, the existent and latent demand for a product that is used due to its high nutritional value; the production and sale costs of the product will let us increase and develop commercial strategies. Peru as the potato origin country, has a great biodiversity of this tuber, producing $72 \%$ of the varieties that exist around the world; the importance of this cultivation at international level is proven, being the fourth basic food in the world population's diet, contributing to reduce the nutritious deficit, in a context where the main goods substitutes show signs of shortage, like it is the case of rice. National Producers of potato are not homogeneous, they vary among the most integrated sector to the markets of goods and factors until the farmers of self-consumption. The interaction between farmers, promoter institutions, investors is needed, The State to develop the profitability and benefits in function of the estimated demand; considering an ideal size, transformation process, market, price, container, promotion of the product and publicity, that is to say, fresh, selected, classified, clean, washed, packed potato and with mark. Taking advantage of the competitive advantages of the Peruvian yellow potato; getting involved national and foreign investors, open to new markets.
\end{abstract}

Key words: yellow potato, offers, it demands, promotion, publicity, profitability.

\footnotetext{
* Este trabajo de investigación fue recibido el 20/06/2009 retornado para su revisión 26/10/2009 y aprobado para su publicación 16/11/2009

1Email:climas@hotmail.com

2Email:jgomezherrera@hotmail.com
} 


\section{INTRODUCCIÓN}

Se recogió información sobre la comercialización de la papa amarilla de la región Junín, dentro del mercado local y regional para lograr identificar y comercializar esta materia prima; se analizó sistemas de demanda latente y cadenas de oferta, costos de producción y venta del producto. Se optimizó las fortalezas que posee este producto, analizando sus debilidades y amenazas dentro de este sistema, identificando el tipo de comercialización dentro del sector agroindustrial.

Ante la necesidad de mejorar la venta como materia prima de la papa amarilla, definiendo un ingreso a mercados más exigentes a nivel local, regional e internacional; se planteó el siguiente problema: ¿Con el estudio de la comercialización en el sector agroindustrial peruano identificaremos la situación actual para mejorar la venta de la papa amarrilla de la Región Junín?

Frente a la interrogante, los objetivos del presente trabajo de investigación fueron: describir el sistema de comercialización actual de la papa amarilla, identificar el procedimiento de mejoramiento para la comercialización correcta de materia prima, y analizar la disposición de materia prima, colocación, sistemas de demanda latente, cadenas de oferta, costos de producción de la papa amarilla.

\section{MATERIAL Y METODOS}

La investigación se realizó en el laboratorio de Ingeniería de los Alimentos de la U.N.C.P. Se utilizó a la papa amarilla con sus variedades más representativas: peruanita, jilguero y tumbay, sembradas en el Valle del Mantaro. Los envases utilizados para la exportación de la papa amarilla preestablecidos son en sacos de $50 \mathrm{~kg}$ y/0 2,5 kg de papas con cáscara, precocidas y congeladas. Estas variedades poseen mayor potencial a nivel internacional, según las exigencias de los consumidores de estos mercados, proponiéndose un tamaño de producto y un peso para su venta, una marca e higiene.
El método de trabajo realizado fue el descriptivo; constó de recopilación bibliográfica sobre la papa amarilla producida en el Valle del Mantaro y en el Perú, se obtuvo datos e información del Ministerio de Agricultura del Perú, Centro Internacional de la Papa, Aduanas, ADEX y otros, sobre la producción, cosecha, post cosecha el estado actual de comercialización y de exportación de la papa amarilla, tipos de mercados, materiales de embalaje; identificando y analizando sistemas de demanda más adecuada y las cadenas de oferta, además de las actividades relacionadas a la penetración y establecimiento de los mercados local, regional e internacional.

\section{RESULTADOS}

La cosecha se efectúa como papa madura para consumo humano. El curado, es para evitar daños de la cáscara y para conservar la papa en buen estado, se debe curar antes de cosecharla, o por lo menos antes de almacenarla; la hace más resistente contra golpes, plagas, enfermedades y secamiento. La temperatura de conservación es de $6^{\circ} \mathrm{C}$ y humedad relativa $85 \%$.

\section{Exportación de papa.}

La exportación de papa amarilla precocida congelada, es la evolución de las exportaciones de papa procesada en el Perú debido a la apertura de la economía peruana y son el resultado de la coincidencia de acciones tanto de empresarios peruanos nacionales privados como de la orientación estratégica de proyectos que aprovechan las ventajas competitivas y comparativas de oportunidad, calidad y exclusividad que tiene el Perú en la producción de papa amarilla. Las exportaciones de papa peruana corresponden fundamentalmente a la procesada. El rubro de exportaciones de papa de mayor importancia en el período 1993-97 en el Perú, fue el de papa cortada en trozos 0 en rodajas, secas, como se indica en el cuadro 1. 
Cuadro 1. Exportaciones de papa procesada (Kg. netos) del Perú años 1993-1997.

\begin{tabular}{ccccccc}
\hline AÑO & $\begin{array}{c}\text { TOTAL } \\
\text { EXPORTACIONES }\end{array}$ & $\begin{array}{c}\text { PREPARADA O } \\
\text { CONSERVADA }\end{array}$ & $\begin{array}{c}\text { CORTADAS } \\
\text { EN TROZOS O } \\
\text { RODAJAS SECAS }\end{array}$ & $\begin{array}{c}\text { HARINA, } \\
\text { FÉCULAY } \\
\text { COPOS DE PAPA }\end{array}$ & CONGELADAS & $\begin{array}{c}\text { PRE COCIDAS } \\
\text { CONGELADAS }\end{array}$ \\
\hline 1993 & 153439 & 10 & 44815 & 27814 & 80810 & 0 \\
1994 & 128508 & 1428 & 86984 & 39507 & 0 & 589 \\
1995 & 100703 & 3257 & 34010 & 31353 & 0 & 32084 \\
1996 & 145864 & 3626 & 26478 & 44230 & 50000 & 21530 \\
1997 & 54443 & 0 & 14389 & 54 & 0 & 40000 \\
Total & 58,958 & 8311 & 206676 & 142958 & 130810 & 94203 \\
\hline
\end{tabular}

Fuente: ADUANAS (1998)

Las tres variedades de papa amarilla peruana más conocidas en el mercado internacional son: "Peruanita" (S. tuberosum ssp. andigena) "Jilguero" u "Ofrenda" (S. goniocalyx); y "Tumbay"; junto con la "Yema de Huevo" de Colombia (S. phureja). Estas variedades de papa son productos nativos de las zonas altoandinas, favorecidas por el microclima de altura (entre los 3200 y 4000 m.s.n.m.), por la radiación solar y por la humedad del ambiente (no más de $65 \%$ de humedad). Al poseer características óptimas para la exportación, se deben realizar campañas de introducción del producto en los Estados Unidos de Norteamérica aprovechando el Tratado de Libre Comercio, ya que es un mercado potencial que permitirá facilitar la participación de empresas peruanas de agricultores, procesadores y exportadores e identificar los canales de distribución empleados por el país importador, mecanismos de abastecimiento y evaluación del comportamiento del mercado.

El programa de "apertura de mercado", campaña de introducción y promoción del producto, debe incluir la producción y difusión, a nivel internacional, de las principales características de la papa amarilla y la edición y distribución de un folleto de carácter promocional, lo cual implica una inversión adicional en productos y servicios.

Los aspectos necesarios a tener en cuenta para entender en qué medida el valor agregado por la transformación es pagado por los consumidores de los países importadores con mercados restringidos, están basados con: ¿cuál es el procesamiento más adecuado? ¿Cuánto cuesta procesar un kilo de papa amarilla? ¿Cómo se forma el precio final de exportación? Para describir el proceso de industrialización de la papa amarilla pelada-precocidacongelada, tomaremos en cuenta la experiencia de procesamiento empleada por la planta de procesados INDICSA para la transformación y empacado de la papa amarilla de exportación "Tambo de Oro" enviada a New York y New Jersey (ver figura 1).

Figura 1. Flujograma del procesamiento de la papa amarilla peladaprecocida-congelada. 
Para establecer la formación de precios se deben considerar todas las etapas producto y los respectivos servicios que intervienen en la exportación del bien final; en este caso desde el abastecimiento de la papa amarilla fresca hasta el traslado al almacén del distribuidor acreditado en el país importador.

Dada la aceptación por parte de los consumidores de un producto final con cáscara, se debe proceder a realizar ensayos para desarrollar el flujo tecnológico: papa amarilla (variedad Peruanita, Jilguero, Tumbay) con cáscara, cocida y congelada. Los resultados de estos ensayos de INDICSA, indicaron que el tamaño ideal de las papas para este proceso, eran las de tamaño mediano (peso por unidad 64,5 g.) y pequeño (peso por unidad 50,5 g.). Además se definió los tiempos y características para la pre-cocción y el congelado IQF. En el caso de la pre-cocción, las de tamaño mediano necesitan una temperatura de 80 a $85^{\circ} \mathrm{C}$ y un tiempo de 25 minutos, mientras que las de tamaño pequeño de $80 \mathrm{a} 85^{\circ} \mathrm{C}$ y un tiempo de 20 minutos. Por el lado del congelado IQF, las de tamaño mediano, necesitan una temperatura de $-30^{\circ} \mathrm{C}$ y un tiempo 12 minutos y las pequeñas $-30^{\circ} \mathrm{C}$ y un tiempo de 8 minutos de proceso.

\section{DISCUSIÓN}

En el Perú existen 2000 variedades de papa y no es posible desarrollar comercialmente todas. La papa amarilla que es oriunda del Perú con sus variedades más representativas: Peruanita, Jilguero y Tumbay, son sembradas en el Valle del Mantaro. Los envases encontrados para la comercialización local son de 100 a $120 \mathrm{~kg}$ con papa sucia, agusanadas podridas, con huecos, que se compran a granel. Para exportación son utilizados sacos de $50 \mathrm{~kg}$ y/0 2,5 kg de papas con cáscara precocidas y congeladas. Por ello debemos promover el desarrollo agroindustrial de la papa, acondicionándonos a las exigencias de un mercado cambiante otorgando al productor posibilidades con un producto "papa fresca, seleccionada, clasificada, limpia, lavada, empacada y con marca”.

La cadena agrocomercial, posee pre-cosecha (preproducción y producción) y post- cosecha (manejo en post-cosecha y mercadeo). Hay que tomar en cuenta el enfoque gerencial de la comercialización que asocia la mercadotecnia a la actividad em- presarial dirigida a utilizar técnicas de investigación de mercados, selección de mercados meta y el posicionamiento. En los mercados existentes y mercados nuevos, para productos nuevos y productos existentes toda empresa debe actuar en cuatro escenarios importantes que son la penetración del mercado, desarrollo del mercado, desarrollo de producto y diversificación.

La participación de una alianza estratégica entre la empresa privada y los proyectos de desarrollo asegurarían la calidad del producto final, manejar un buen sistema de producción en campo, adecuados canales de comercialización. Un sistema de asistencia técnica desde la multiplicación de semilla hasta la producción comercial, asegura el volumen de ventas, que es un indicador del éxito de comercialización. Se debe integrar la exportación de papa amarilla en su estrategia de apoyo al pequeño productor pobre de la Sierra peruana, como del Valle del Mantaro, por ser un "producto único" en el mundo, debido al microclima, altura, radiación solar y humedad del medio ambiente donde se produce. Adoptar un conjunto de acciones de desarrollo tecnológico y generar valor agregado que reduzca los precios unitarios, hasta hacer competitivos los precios de la papa amarilla importada dentro del mercado norteamericano y otros.

Con el proceso de liberalización de la economía, iniciado en 1990, el Perú ha empezado la búsqueda de mercados para sus papas nativas. En los primeros años de la década 1980 - 1990 ya existían firmas exportando este tubérculo, dentro de una mezcla de productos nativos peruanos enteros, precocidos y congelados.

Dentro de los principales resultados entre distribuidores, gerentes de supermercados y representantes de cadenas en el extranjero, en términos de las ventajas y desventajas del producto, fueron que los compradores están interesados en nuevos productos "gourmet"; éstos sólo negocian a través de sus distribuidores de confianza que presentan el producto, definen precios y volúmenes, realizando la promoción, degustación y publicidad del producto. Quieren contar con un surtido de productos que incluya, además de papa amarilla, cereales, camarones, café y cacao, etc. Hay algunas cadenas de autoservicios que no promueven el ingreso de nuevos productos, sólo se 
interesan si el producto ya está comercializado por lo menos en una cadena. Todos los compradores piden que se les garantice una oferta continua durante todo el año, lo que implica incorporar otros valles productores del Perú en papa amarilla con diferentes calendarios de cosecha.

Cuadro 3. Modelo de fase de introducción de papa amarilla a exportar.

\begin{tabular}{|c|c|c|c|c|}
\hline & $\begin{array}{l}\text { Agricultores del } \\
\text { Valle del } \\
\text { Mantaro }\end{array}$ & $\begin{array}{l}\text { Mercado } \\
\text { mayorista }\end{array}$ & $\begin{array}{l}\text { Mercado } \\
\text { minorista }\end{array}$ & Consumidores \\
\hline PRODUCTO & $\begin{array}{l}\text { Papa } \\
\text { seleccionada } \\
\text { (sacos } \\
\text { normales) }\end{array}$ & $\begin{array}{l}\text { Papa } \\
\text { seleccionada } \\
\text { limpia y } \\
\text { envasada, } \\
\text { marca, rótulo en } \\
\text { envase de } 50 \mathrm{~kg}\end{array}$ & $\begin{array}{l}\text { Papa } \\
\text { seleccionada, } \\
\text { limpia y } \\
\text { envasada, marca }\end{array}$ & $\begin{array}{l}\text { Papa de buena } \\
\text { calidad }\end{array}$ \\
\hline PRECIO & $\begin{array}{l}\text { Aumento por } \\
\text { mejor selección }\end{array}$ & $\begin{array}{l}\text { Procesamiento } \\
\text { Nuevo empaque }\end{array}$ & $\begin{array}{l}\text { Procesamiento } \\
\text { Nuevo empaque }\end{array}$ & $\begin{array}{l}10 \text { a } 15 \% \\
\text { adicional por } \\
\text { producto de } \\
\text { mayor calidad }\end{array}$ \\
\hline PLAZA & $\begin{array}{l}\text { Mercado } \\
\text { mayorista }\end{array}$ & $\begin{array}{l}\text { Comerciantes } \\
\text { minoristas }\end{array}$ & $\begin{array}{l}\text { Mercados de } \\
\text { abastos }\end{array}$ & $\begin{array}{l}\text { Mercados de } \\
\text { abastos }\end{array}$ \\
\hline PROMOCIÓN & $\begin{array}{l}\text { Asistencia } \\
\text { Técnica }\end{array}$ & $\begin{array}{l}\text { Diferenciación } \\
\text { de producto y } \\
\text { empaque (Color } \\
\text { e información) }\end{array}$ & $\begin{array}{l}\text { Diferenciación } \\
\text { de producto + } \\
\text { publicidad } \\
\text { (afiches, } \\
\text { trípticos) }\end{array}$ & $\begin{array}{l}\text { Anuncio de } \\
\text { presencia del } \\
\text { producto }\end{array}$ \\
\hline
\end{tabular}

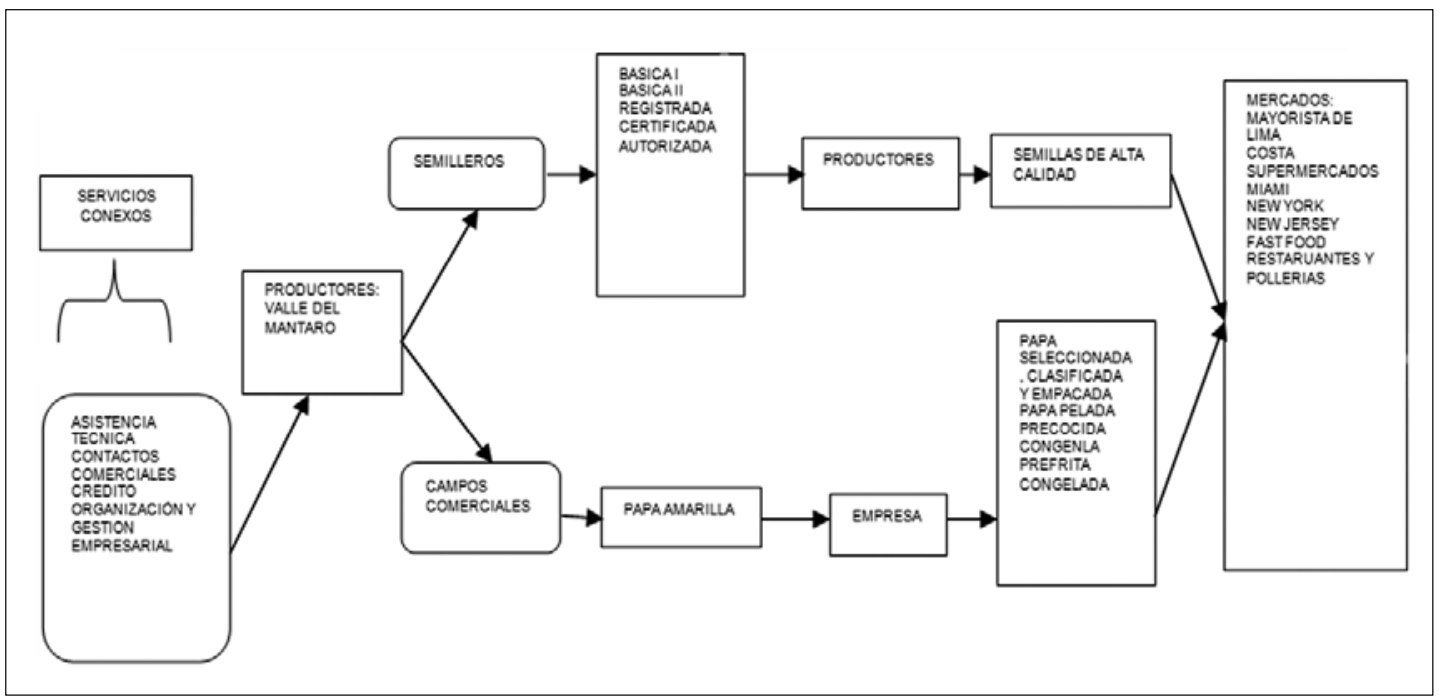

Figura 3. Esquema de articulación de papa amarilla. 


\section{CONCLUSIONES}

- Existe interés en el mercado internacional por productos procesados de papa, particularmente por la papa amarilla, debido a su calidad y sabor, presenta mucha expectativa hacia la papa amarilla proveniente de Perú, especialmente precocida y congelada.

- El aprovechamiento de las ventajas competitivas y comparativas de la papa amarilla peruana, requieren el desarrollo de un mercado más amplio en la búsqueda de precios competitivos. En todas las experiencias de introducción de un producto nuevo en el mercado, una de las limitantes del consumo es el elevado precio relativo en comparación con las papas producidas internamente 0 importadas de otros países.

- Debemos interesar a inversionistas y socios potenciales para participar en el mercado, enfrentando una inversión conjunta que se oriente a desarrollar el nivel de rentabilidad y beneficios, en función del tamaño de demanda estimada; con el estudio de la demanda.

- La apertura de mercados es un proceso complejo y laborioso, donde intervienen varios agentes económicos tanto del país exportador como del importador, y se requiere negociar todos estos intereses en función de un solo objetivo.

\section{LITERATURA CITADA}

Alap. 2001. Revista latinoamericana de la papa. Lima Perú. 100 pág.

Alarcón, j. 2002. Mercadeo de productos agropecuarios. Edit. A4 impresores S.R.L. Lima Perú. 375 pág.

Alvarado, j. Y aguilera, j. 2001. Métodos para medir propiedades físicas en industrias de alimentos. Edit. Acribia s.a. Zaragoza España. 250 pág.

Centro internacional de la papa. 2008. Producción de la papa en la región Junín. Cip. Huancayo Perú. 30 pág.

Cheftel, j. 1990. Introducción a la bioquímica y tecnología de los alimentos, Edit. Acribia S.A. Zaragoza España. 250 pág.

Cuánto. 2008. Perú en números 2007. Edit. Cuánto. Lima Perú. 1294 pág.

Tapia, m. Y fries, a. 2007. Guía de campo de los cultivos andinos. Fao, Anpe, Lima Perú. 209 pág.

Tocagni, h. 1980. Producción de papas. Edit. Albatros. Argentina. 171 pág. 